\title{
Essais
}

Revue interdisciplinaire d'Humanités

Narration et lien social

\section{Ilya Kabakov, ou le récit de vies communautaires}

\section{Alice Cazaux}

\section{OpenEdition}

Journals

Édition électronique

URL : https://journals.openedition.org/essais/9299

DOI : 10.4000/essais. 9299

ISSN : 2276-0970

\section{Éditeur}

École doctorale Montaigne Humanités

\section{Édition imprimée}

Date de publication : 15 mai 2013

Pagination : 101-120

ISBN : 978-2-9544269-0-7

ISSN : 2417-4211

\section{Référence électronique}

Alice Cazaux, "llya Kabakov, ou le récit de vies communautaires », Essais [En ligne], 3 | 2013, mis en ligne le 01 septembre 2021, consulté le 08 septembre 2021. URL : http://journals.openedition.org/ essais/9299 ; DOI : https://doi.org/10.4000/essais.9299 


\section{Ilya Kabakov, ou le récit de vies communautaires}

\section{Alice Cazaux}

Ilya Kabakov célèbre le non-mémorable en confisquant aux faiseurs d'histoire leur manière de dire et de figurer, leur fausse grandeur et leurs chants hérö̈ques. Il leur fait la peau et la retourne en un tour de main pour montrer la chair contrainte et ses palpitations.

François Barré

Ilya Kabakov, qui se qualifie lui-même d'artiste soviétique, a longtemps été illustrateur de livres pour enfants et membre de L'Union des Artistes en URSS. En parallèle, il était l'une des figures de proue du mouvement non-officiel moscovite. Sa pratique s'ancre profondément dans la narration du monde, absurde et poétique, de la période qui l'a engendré. Ainsi, dans des installations totales, qui englobent le spectateur jusqu'à le transformer en acteur, il tisse la toile d'un récit surréaliste du socialisme. Au fil de ses œuvres, il n'esquisse pas le caractère politique qu'on voudrait lié à la représentation de l'URSS, mais il suggère le récit d'un "vivre ensemble " forcé ; des personnages absents, fantomatiques, mais dont l'histoire donnée à lire dans l'œuvre même porte les stigmates de la vie communautaire, et des oppositions qui la caractérisent : public/privé, discours/pensée, réel/imaginaire.

L'étude de quelques-unes de ces installations permettra d'envisager la place qu'accorde cet artiste au lien entre récit et création plastique, recréant ce lien social si violent, et rendant ses œuvres " plus vraies que nature ». N'écrit-il pas lui-même : «Lappartement communautaire, pour moi, au moins (mais pour les autres aussi, je pense) est sans aucun doute l'endroit, l'espace, qui démontre de manière exemplaire l'effet que produit sur un être humain un lieu d'habitation spécialement agencé, semble-t-il, pour mettre à exécution le verdict : "l'enfer, c'est les autres" " ?

1 Ilya Kabakov, in Ilya Kabakov Installations 1983-1995, Paris, éd. du Centre Georges Pompidou, 1995 , p. 68. 
Nous verrons par quels moyens il narre les résistances et les conflits liés à cette vie en kommunalka (une famille par chambre, partage des parties communes), quels sont les outils de création d'un imaginaire à valeur de témoignage.

\section{Rappel biographique}

Ilya Kabakov est né en 1933 à Dniepopetrovsk, actuelle Ukraine. Il connaîtra une première migration dans les années 1940 jusqu’à Samarkand en Ouzbékistan. De parents juifs, il y est exilé avec sa mère pour fuir les nazis alors que son père part au front. Malgré des conditions de vie et de travail difficiles pour les réfugiés, dès l'âge de dix ans il intègre l'École d'Art de Leningrad, elle aussi évacuée à Samarkand. Il étudiera ensuite au sein d'un collège spécialisé dans les arts à Moscou, puis à l'Institut Surikov, d'où il sera diplômé en 1957, se liant à ceux qui formeront l'intelligentsia artistique moscovite.

À 20 ans, il devient illustrateur de livres pour enfants, mais ne s'épanouissant pas dans le réalisme socialiste ou la reproduction des classiques russes, il élabore un style personnel, et commence à mener en contrepoint une pratique non-officielle ${ }^{2}$ - abstraction, puis conceptualisme. Toutes les expositions de ce petit cercle des conceptualistes moscovites sont privées, se déroulent en appartement pour de très brefs délais, souvent le temps du vernissage, et les œuvres sont décrochées et démontées après l'événement.

Grâce à la visite de critiques venus de l'ouest, dans les années 1980 son travail est reconnu à l'étranger, et il est invité à exposer hors des frontières de l'URSS. Mais à la différence d'autres artistes non-officiels, son travail d'illustrateur permet à Ilya Kabakov de devenir membre de l'Union des Artistes ${ }^{3}$. Il a donc accès à du matériel, un atelier, et perçoit des cachets pour ses illustrations. Ses relations avec la sphère officielle ne sont pas aussi frontales et provocatrices que semble l'indiquer le titre de "non-officiel ».

Son passage à l'ouest s'est déroulé en plusieurs étapes. À partir de 1985, il est reconnu en Europe grâce aux expositions qui lui sont successivement consacrées à Berne, Marseille, Düsseldorf et Paris ${ }^{4}$. En 1988, il réalise sa première installation totale à la galerie Ronald Feldman, New-York ${ }^{5}$ Les Dix Personnages. Il

2 Il semble important de relever une différence primordiale entre « art dissident " et " art nonofficiel ». L'art dissident est profondément politique et dirigé à l'encontre des directives du Parti, alors que l'art non-officiel l'est par défaut, aucune institution ne permettant de rendre sa pratique et son exposition « officielles".

3 À partir de 1965.

4 En 1985 lui sont consacrées deux expositions personnelles : "Along the Margins ", à la Kunsthalle de Berne en Suisse, qui voyagera à Marseille puis Düsseldorf en Allemagne, puis "Kabakov » à la Galerie Dina Vierny, Paris.

5 Décidément très novatrice, puisque c'est elle qui avait accueilli la première exposition internationale de Komar et Melamid en 1976. 
passera deux ans à Berlin, bénéficiaire d'une bourse de création, puis travaillera chez Dina Vierny ${ }^{6}$ avant d'être professeur invité de l'école des Beaux-arts de Francfort. Il s'établira à New-York en 1992 avec son épouse et collaboratrice Emilia, partageant leur temps entre les États-Unis et les lieux d'exposition.

En 2004, une importante exposition leur été consacrée au musée de l'Ermitage à Saint-Pétersbourg, signe de la reconnaissance institutionnelle russe affirmée envers ce travail d'envergure.

\section{Les kommunalki et l'œuvre d'art totale}

\subsection{Le phénomène}

La vie en kommunalka, ou appartement communautaire, perdure en Russie depuis près d'un siècle et concerne encore des milliers de Russes, qui vivent dans l'astreinte d'une cohabitation imposée alors qu'aucune attache familiale ou sociale ne les lie.

Les "maisons communes " sont apparues en 1917 en Russie. C'est au départ un idéal bolchévique imaginant la construction d'habitations communes en vue d'un nivèlement social : des logements permettant à toutes les classes de se côtoyer au quotidien. Mais en 1918 le pouvoir commença à réquisitionner les hôtels particuliers et les maisons bourgeoises pour y loger les travailleurs arrivés en ville, dans un plan de "densification " des lieux déjà existants, s'éloignant de la construction d'habitats utopiques. Cet idéal devint un palliatif, celui de la crise du logement urbain provoquée par l'exode rural.

"Remplissage ", " ouplotnienie " en russe, voilà le terme extraordinaire que les
communistes ont utilisé pour définir la gigantesque opération qui a consisté à
installer dans un appartement autant de familles qu'il comptait de pièces, sans
jamais tenir compte ni de leur origine, ni de leur métier, ni de leur histoire, au
nom du nivellement social et du manque d'espace. Pour les dirigeants et pour
Lénine en premier lieu, c'était à la fois une solution à la crise du logement et
une conquête idéologique. "Eh bien, messieurs, qu' est-ce donc que ces appar-
tements luxueux, c'est honteux, vous devez les partager avec les travailleurs ",
disaient les bolchéviques.

Les propriétaires se virent ainsi amputer de quelques pièces, puis bientôt de leurs droits à la propriété. En 1918, c'est l'État qui devint seul gestionnaire du patrimoine immobilier national. ${ }^{8}$ La délation y est prévue dès l'origine :

6 C’est au Musée Maillol - Fondation Dina Vierny, Paris -, qu'est exposée de manière permanente son installation La Cuisine communautaire, 1991.

7 Témoignage collecté par Paola Messana, Kommunalka, Une histoire de l'Union soviétique à travers les appartements communautaires, J.-C. Lattès, 1995, p. 14.

8 "...] après plusieurs arrêtés au cours du mois de septembre 1918, le Soviet publie le $1^{\text {er }}$ novembre un arrêté général "Sur la municipalisation des habitations et des terrains " qui reprend, précise ou modifie les directives précédentes. Cet arrêté supprime définitivement le droit à la propriété 
Lénine avait chargé le Commissariat du peuple aux affaires intérieures, le NKVD, de trouver des indicateurs rémunérés parmi les habitants.?

Dans les années 1930, de vastes programmes de construction d'immeubles furent imaginés mais jamais réalisés, conférant à la kommunalka sa place d'habitat majoritaire, " avec son mode de vie, ses conflits, sa saleté, ses parties communes dégradées... ${ }^{10}$. Ce qui n'était plus un projet idéologique mais une solution temporaire s'ancra finalement dans le temps et la société soviétiques.

Après la Grande Guerre Patriotique, la seconde Guerre Mondiale, une campagne de construction de logements "mono-familiaux " vit le jour, dans une tentative de distribution d'appartements individuels :

Les nouvelles "Normes et règles de construction ${ }^{11}$ du $1^{\text {er }}$ mars 1958 déterminent l'unique mode d'attribution du logement : une famille par appartement, mais donnent un sursis au mode communautaire, perpétué dans des appartements de six pièces de 9 à $15 \mathrm{~m}^{2}$ chacune, "pour célibataires et petites familles ». ${ }^{12}$

Khrouchtchev fit à son tour construire des tours d'immeubles à habitations mono-familiales en périphérie des grandes villes pour réduire la proportion de kommunalki. Ces appartements, construits dans l'urgence avec des matériaux bon marché, n'étaient pas du meilleur confort. Ainsi, ils donnèrent lieu à l'invention du mot "khrushchoba ", association du nom de Khrouchtchev, "Khrushchev ", avec le mot taudis, " trushchoba ». ${ }^{13}$

Le manque de logement contribua au maintien des appartements communautaires. Pour éviter l'engorgement des listes d'attente, l'accès à la propriété était refusé aux célibataires et aux retraités. C'est pourquoi aujourd'hui encore, la grande majorité des occupants de kommunalki sont des personnes seules, souvent invalides, âgées ou marginales, ce qui pose des problèmes d'ordre tant sanitaire que social.

La ville de Leningrad (aujourd'hui Saint-Pétersbourg) fut celle qui accueillit le plus grand nombre d'appartements communautaires, et c'est toujours le cas. On l'appelle gorod kommunalok, la «ville des kommunalki».

privée sur les biens immobiliers. ", in Katarina Azarova, L'appartement communautaire - L'histoire cachée du logement soviétique, éd. du Sextan, Paris, 2007, p. 149-150.

9 Paola Messana, op. cit., p. 11.

10 Géraldine Pavlov, "Kommunalka : l'enfer, c'est les autres... ", revue en ligne Regards sur l'Est, janvier 2006 (ISSN 2102-6017) : http://www.regard-est.com/home/breve_contenu. php?id=557.

11 Stroitelnye normy i pravila (Les Normes et les règles de la construction). SNIP II. B 10-57, in Katarina Azarova, L'appartement communautaire - L'histoire cachée du logement soviétique, Paris, éd. du Sextan, 2007.

12 Ibid., p. 203-204.

13 Katarina Azarova, "La "question du logement", l'appartement communautaire et la privatisation de l'habitat à Moscou ", Revue d'études comparatives Est-Ouest, Les villes russes après une décennie de réformes, vol. 32, 2001, nº 4, p. 185-216, p. 199. 
Les chiffres officiels (par ailleurs souvent mensongers) ne prennent pas en considération la différence entre la situation moyenne dans le pays et celle dans les villes, notamment à Moscou. Toutefois, certaines données peuvent être révélatrices. Ainsi, d'après le recensement de 1989, les 89,5 millions de ménages que comptait le pays ne disposaient que de 51016 millions de logements. Selon les calculs de Michèle Kahn (1992), basés également sur des chiffres officiels, environ $45 \%$ de la population des villes auraient eu besoin d'un logement en $1990 .^{14}$

Au moment de la fragmentation et de l'effritement du bloc communiste dans les années 1990, ces appartements furent privatisés en grand nombre. Des agences immobilières en relogeaient les occupants avant de vendre l'appartement dans son ensemble à un particulier, appartenant généralement à cette classe naissante qualifiée par le terme de nouveaux riches. De même, les locataires pouvaient devenir propriétaire de leur chambre. Il était fréquent que les pressions sur les habitants leur fassent quitter les lieux sous la menace d'agents immobiliers aux principes discutables, qui le rachetaient à prix avantageux. Grâce à des plans d'urbanisme à grande échelle et à une " décommunautarisation " des appartements favorisée par le gouvernement, la situation tend à s'amenuiser :

Tout cela a contribué à réduire considérablement le nombre d'appartements communautaires qui, en avril 1998, ne représentaient plus que 3,5\% du total des logements à Moscou ${ }^{15}$ (contre $40 \%$ au début des années 1980 $\left.{ }^{16}\right) .{ }^{17}$

Mais à Saint-Pétersbourg, en 2005, $70 \%$ des appartements du centre ville sont communautaires et abritent 536000 personnes (11\% de la population). ${ }^{18}$

Ce problème est encore très présent dans la mémoire collective, et souvent dans la vie quotidienne des habitants des grandes villes.

\subsection{Représentations de l'univers soviétique}

Depuis les années 1970 en Russie, différents modes de représentation du monde soviétique ont été utilisés par les artistes. La plus remarquable a posteriori est certainement le Sots Art, contraction des mots sotsialist et art, terme

14 Katerina Azarova, «La "question du logement", l'appartement communautaire et la privatisation de l'habitat à Moscou ", op. cit., p. 187.

15 D'après les données de l'inventaire réalisé par la municipalité de Moscou, en février-avril 1998, dans le cadre de la préparation du "Programme de liquidation des logements communautaires ".

16 Ce chiffre provient de l'Annuaire statistique de l'URSS pour l'année 1980 et représente le pourcentage moyen pour l'ensemble des villes du pays. Ce problème se posant toujours avec plus d'acuité à Moscou et Leningrad que dans les autres villes, on peut donc supposer que le nombre de logements communautaires y était encore plus élevé.

17 Katerina Azarova, «La "question du logement", l'appartement communautaire et la privatisation de l'habitat à Moscou ", op. cit., p. 202.

18 Géraldine Pavlov, op. cit. 
utilisé pour décrire le pop art russe. Des artistes comme Komar et Melamid, Leonid Sokov, Alexander Kosolapov, et d'autres encore utilisaient les codes mêmes du réalisme socialiste pour les court-circuiter. Ils en empruntaient les signes mais altéraient les signifiés, discréditant ainsi les images sinon sacrées du moins sacralisées des dirigeants, des héros - figures de proue de l'imagerie soviétique tels l'ouvrier et la kolkhozienne - et des différents thèmes abordés par cet art propagandiste de masse. En d'autres termes, ils utilisaient les images omniprésentes dans l'univers soviétique pour les détourner et faire surgir l'insensé, alors comique ou en manque de sens.

A contrario, s'il a participéà la cristallisation de ce mouvement, Ilya Kabakov ne brouille aucune piste. Il ne s'engage pas dans un détournement des représentations à travers ses installations totales, mais dans une présentation subjective d'un monde investi par les objets. Les objets sont ce qu'ils sont, et le sens auxquels ils renvoient ne s'en éloigne en rien. L'objet et le texte sont en totale complémentarité.

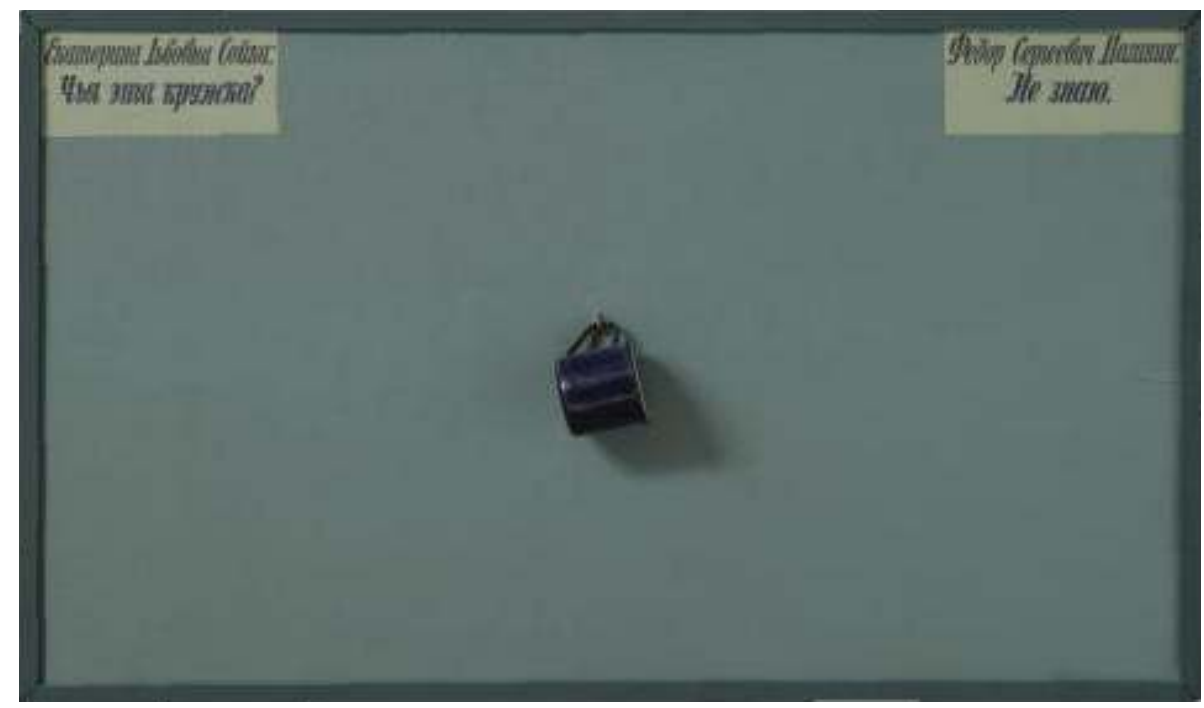

Ilya Kabakov, Ekaterina Lvovna Sö̈ka : À qui est cette tasse ?, 1991.

Par exemple, près d'une tasse nous pouvons lire : "À qui est cette tasse ? ". Tout de cette tasse, jusqu'à la peinture du tableau sur laquelle elle est clouée, renvoie à l'univers soviétique ${ }^{19}$. D'un objet purement fonctionnel l'artiste organise un tableau, de valeur subjective ou inessentielle. Grâce au texte, grâce à ce questionnement d'une potentielle propriété, c'est cette dernière valeur qui prime, rendant à l'objet son univers et lui inventant un absolu.

19 Les murs intérieurs des bâtiments soviétiques, et c'est une habitude qui perdure, étaient souvent peints jusqu’à mi-hauteur en gris, brun ou vert. 
Ce tableau fait écho à la chaise conceptuelle de Kosuth, 1965 : la chaise, son image et sa définition. Ici, nous avons la tasse, son propriétaire fictif, et l'absurdité du dialogue amenée par la réponse : "Je ne sais pas ». Ce questionnement sur la propriété est un indice important du manque de communication relayé dans l'imaginaire des kommunalki. Si l'on demande à qui est cette tasse on peut penser qu'elle n'est pas à sa place, c'est-à-dire dans l'univers délimité de son propriétaire : sa chambre - espace privatif de l'appartement commun - ou son placard - espace privatif de la cuisine commune.

Le questionnement a ici valeur de définition de l'univers soviétique. Chez Ilya Kabakov, les objets sont l'incarnation du temps et de l'espace duquel ils sont issus, et en diffusent l'aura. L'association objet-texte crée un ensemble qui permet de conter l'inénarrable.

Les appartements communautaires apparaissent en forts espaces symboliques, qui concentrent les problématiques du vivre ensemble soviétique :

- La différence des milieux professionnels et des sujets de préoccupation des habitants du même appartement.

- La différence des usages en ce qui concerne le maintien de l'ordre et de la propreté.

- La cohabitation dans la même pièce des enfants qui grandissent avec les parents, et des filles avec les garçons.

- La cohabitation dans le même appartement, et parfois dans la même pièce, des étudiants et des travailleurs.

- La nécessité de passer chez les voisins pour entrer chez soi, surtout dans la soirée ou dans la nuit, et d'autres inconvénients dus au dispositif spatial adapté à une seule famille.

- L'animosité entre les différents habitants du même appartement, souvent accompagnée de querelles quotidiennes.

Tous ces faits sont devenus habituels et ont des conséquences très graves pour la santé physique et mentale des habitants, ainsi que pour la qualité de l'éducation. ${ }^{20}$

Ainsi, Ilya Kabakov, pour traduire et conter, faire vivre cette expérience, en appelle parfois à la kommunalka :

[...] je compris que l'histoire de l'URSS, de son idéologie, de son peuple, de ses comportements humains qui souvent nous déroutent se reflétait dans ces appartements, lorsqu'elle n'en était pas le fruit. ${ }^{21}$

L'appartement communautaire est le décor dans lequel l'artiste peut nous présenter une multitude de personnages et de situations fictives ou vécues, donnant à voir un échantillon de la société soviétique, expérimentée alors subjectivement par le regardeur.

20 S. I. Snejder, Les Maisons-Communes du quartier Krasnopresnenskij, Moscou, 1925, in Katerina Azarova, "La "question du logement", l'appartement communautaire et la privatisation de l'habitat à Moscou ", op. cit., p. 194-195.

21 Paola Messana, op. cit., p. 8. 


\subsection{L'Installation Totale}

Une représentation si complète de cet univers est rendue possible grâce au concept d'installation totale, qui permet un lieu de confrontation des subjectivités. Ilya Kabakov a inventé ce concept comme étant la fusion en un seul lieu de divers média : peinture, sculpture, écriture, musique, sons, voix ou chant. Les textes y ont une place particulière :

[Ils] ne sont pas simplement des éléments de langage appliqués aux objets visuels, dans un quelconque sens profondément didactique, ils y amènent un sens radicalement nouveau. Le plus souvent, les textes présents dans les installations sont des extraits, ou des condensés des propres textes et réflexions théoriques de Kabakov, parfois collectés par une tierce personne. En tant que partie intégrante de l'installation "totale ", ils remplissent une fonction complexe de commentaire.

[...] Kabakov attribua à la « capacité " de l'installation « totale » d'intégrer non seulement des éléments des beaux-arts - dessin, peinture, objets - mais aussi des emprunts à d'autres genres, comme la littérature, la musique et le théâtre, et de les combiner à la manière du Gesamtkunstwerk. ${ }^{22}$

Gesamtkunstwerk est le terme wagnérien de désignation d'une œuvre unissant tous les arts dans une dynamique de révolution sociale et esthétique. Avec comme modèle la tragédie antique, Wagner théoricien aspire à une révolution de l'homme, de la société et à travers elle de son $\operatorname{art}^{23}$.

Lorsque Ilya Kabakov utilise le terme d'installation totale, il est assez clair qu'il fait référence à la définition wagnérienne. Mais il faut comprendre cette citation dans ce qu'elle a d'esthétique, et non de politique : ce n'est pas dans un désir de révolution que l'installation est totale, mais dans celui de synthèse des pratiques plasticiennes (différentes techniques, divers média et œuvres) en un même résultat, une même installation.

Entre cette redoutable utopie [de l'idéal d'un art salvateur, capable de rétablir ou de recréer l'unité perdue (métaphysiquement ou socialement)] et la banale association des arts, l'aspiration à l'œuvre d'art totale est susceptible de multiples degrés et de très diverses interprétations. ${ }^{24}$

22 Tony Stoos, in Ilya Kabakov : Installations. Catalogue Raisonné 1983-2000, Tony Stoos ed., Allemagne, Richter Verlag éditions, 2003, t. I, p. 9-10. Traduction personnelle de l'anglais.

23 Richard Wagner, L'Euvre d'art de l'avenir, réédition conforme au retirage de 1928 de l'Édition Delagrave, Paris, 1910. Traduction en français par J.-G. Prod'homme et Fritz Holl, collection Les Introuvables, Éd. d'Aujourd'hui, 1982, p. 61 :

"L'homme ne sera pas ce qu'il peut et doit être tant que sa vie ne sera pas le miroir fidèle de la nature (...) C'est alors seulement que l'homme sera en réalité homme, tandis que, jusqu'à présent, il n’a jamais existé qu'en tant qu'attribut tiré de la religion, de la nationalité ou de l'État. De même, l'art ne sera pas ce qu'il peut et doit être, tant qu'il ne sera pas l'image fidèle du véritable homme et de la véritable vie nécessaire aux hommes, donc, tant qu'il lui faudra emprunter les conditions de son existence aux erreurs, travers et monstruosités de notre vie moderne. »

24 Jean Galard et Julián Zugazagoitia, in L'ouvre d'art totale, collection Art et artistes, éditions du Louvre et Gallimard, 2003, p. 6. 
Un de ces degrés est l'installation : considérons que le caractère total des installations d'Ilya Kabakov est avant tout un genre qui synthétise plusieurs formes d'art et plusieurs œuvres, n'ayant en aucun cas une aspiration révolutionnaire.

Ainsi, selon Boris Groys, " notre monde entier est une vaste installation... une installation divine ${ }^{25}$. Sans pour autant aller jusqu'à Dieu, nous pouvons constater que l'artiste Ilya Kabakov crée une image du monde, ou plutôt « un modèle de monde $»^{26}$. Ses installations sont souvent construites de manière à englober le spectateur, qui peut éprouver l'œuvre à la manière d'un monde parallèle. Il est conscient de l'existence du monde extérieur, mais joue à s'imaginer et se mouvoir, se vivre dans un monde qui lui est proposé.

L'installation totale est un environnement dans lequel le spectateur peut évoluer, guidé par le parcours proposé par l'artiste (entrée-sortie), mais dans l'autonomie de ses choix de lecture, de ses intérêts propres pour l'une ou l'autre des parties de l'installation.

Ce sont $[\ldots]$ deux possibilités d'avenir historique que Kabakov met en scène dans ses installations, qui sont presque toujours construites de sorte que qu'une partie se trouve bien éclairée, accessible et visible, cependant qu'une autre pâtit d'un mauvais éclairage ou demeure difficilement accessible. Ailleurs, Kabakov use d'autres procédés, comme, par exemple, l'exploitation de l'ennui qui, avec le temps, s'empare immanquablement du spectateur. Cette fatigue l'empêche de continuer à regarder les images et à lire les textes, abolissant ainsi la frontière nette entre le vu et le non vu. ${ }^{27}$

Des ombres resteront au tableau - le spectateur lambda ne pourra tout lire, tout identifier, tout expérimenter. Ilya Kabakov place délibérément trop de textes à lire, des objets dans des zones d'ombre, des éléments qui, selon les spectateurs, resteront invisibles. C'est une forme d'expérimentation de l'art qui se rapproche de la vie, laissant au spectateur le choix de son œuvre ; c'est une forme totale et absolue dont le regardeur ne pourra saisir qu'une partie, subjective.

\section{Les Dix Personnages}

Exposée pour la première fois à la galerie Ronald Feldman, New York, en 1988, cette installation doit être l'une des plus célèbres de l'artiste. Elle est consacrée à la description de personnages tous atteints d'une folie plus ou moins douce. Voici le décor posé par l’artiste :

25 Boris Groys, in Oskar Bätschmann, «Ilya Kabakov and the "Total” Installation », in Ilya Kabakov: Installations. Catalogue Raisonné, op. cit., p. 20.

26 Oskar Bätschmann, ibid.

27 Boris Groys, «Un homme qui veut duper le temps », in Ilya Kabakov Installations 1983-1995, op. cit., p. 19. 
L'installation comprend les dix pièces des dix personnages, ainsi que celle du locataire qui a déménagé [...], et le couloir qui les sépare. Situées de part et d'autre de celui-ci et séparées par des cloisons, les chambres sont des renfoncements sans fenêtres [...] Dans chaque pièce, il reste quelques objets appartenant au personnage. On y trouve aussi un dépliant qui explique son histoire et son projet personnel. Le couloir et les chambres sont éclairés si parcimonieusement, par de petites ampoules suspendues au plafond, qu'on parvient à peine à lire $[. .$.

Voici la liste des « habitants » de cette installation, que leur surnom caractérise clairement :

- L'homme qui s'est envolé dans son tableau.

- L'homme qui collectionne les opinions des autres.

- L'homme qui s'est envolé dans l'espace.

- L'artiste sans talent.

- L'homme tout petit.

- Le compositeur.

- Le collectionneur.

- L'homme qui se décrit à travers d'autres personnages.

- L’homme qui sauve Nikolaï Viktorovitch.

- L'homme qui ne jetait jamais rien. ${ }^{28}$

Le couloir de l'appartement communautaire intervient comme la structure narrative de l'œuvre. Il faut ajouter à cela les textes présents dans chaque installation, relatant l'histoire de chaque habitant. Ces personnages imaginaires trouvent leur source dans une autre structure narrative : un des premiers projets d'envergure de Kabakov, ses Albums, source génétique des installations qui suivirent. On y retrouve plusieurs thèmes récurrents, notamment l'envol, l'artiste incompris ou la mouche. Ces Albums, et les personnages que Kabakov y a fait naître, seront progressivement intégrés, parfois dans leur forme même, aux installations et aux futurs travaux de l'artiste.

\subsection{Les Albums, ou la genèse perpétuelle}

Ilya Kabakov partagea son statut d'illustrateur avec Erik Bulatov notamment, qui pratiquait lui aussi une activité artistique parallèle et non officielle. De cette pratique de l'illustration, bidimensionnelle, avec un temps donné (celui du page-après-page), la mise en espace se passa progressivement. Les Albums en sont la première étape, consacrés chacun à un personnage dont l'histoire personnelle - à rapprocher de celle du héros du roman russe, un petit homme incompris de la masse - est contée à travers une architecture de textes et d'images. L'atelier du Stretenski boulevard à Moscou accueillait les amis de l'artiste, qui feuilletaient les pages de ces histoires inventées se découvrant dans l'univers très privé - et privilégié - de l'atelier. 
Par la suite, l'artiste développa des expositions éphémères, présentant ses travaux sur des dépliants, apparaissant à la frontière entre un travail d'illustration plane et une mise en espace de la narration.

Kabakov, dans ses Albums, récupère des créations éparses qu' il fait fonctionner ensemble. Il associe par exemple un dessin à un texte, puis y ajoute un dessin des années après. Il est dans une refonte permanente de son travail plastique, refonte qui perdure avec la forme des installations, particulièrement propice à ce procédé. Chaque œuvre nouvelle est constituée en partie d'anciens travaux :

Kabakov a toujours systématiquement récapitulé et réécrit son passé à neuf. Ainsi, après avoir développé la forme des Albums au début des années 1970, il y a intégré ses anciens dessins. [...] Cette "mort " les a sauvés du dépassement du temps et leur a permis d'être intégrés dans un contexte nouveau et actuel. Kabakov procède de la même manière avec ses tableaux anciens, qu'il intègre, encore et toujours, à des installations construites beaucoup plus tard. ${ }^{29}$

Si les Albums sont effectivement à considérer comme un travail de genèse - constituant, bien entendu, une ouvre à part entière - il faut considérer ce remaniement permanent du travail de l'artiste comme une de ses caractéristiques principales. Ainsi, nous pouvons parler de réécriture continue de son corpus, et abolir la frontière entre écriture, illustration et installation, puisque ces trois média constituent l'œuvre - narrative - dans son intégralité.

Notons également que les Albums ont été créés en période de pénurie (peu de matériaux, d'espaces d'exposition, de libertés). Ce n'est qu'en Occident, accompagné d'une reconnaissance publique assortie d'une abondance de matériaux, d'espace et de libertés, que l'artiste a pu donner vie à des idées couchées sur le papier, isolées ou reléguées dans ses archives personnelles. L'installation totale est aussi le moyen pour Kabakov d'intégrer les thématiques de ses Albums, et de leur donner vie à la manière d'un livre animé - pop up - qui déplie ses décors à mesure qu'on en tourne le pages. Ainsi, Les Dix Personnages est une installation tirée de la série de dix albums du même titre, réalisés entre 1972 et 1975. De même, elle englobe en son sein plusieurs installations déjà existantes : L'Homme qui s'est envolé dans son Tableau (1981), et L'homme qui sest envolé dans l'espace (1985). L'homme qui ne jetait jamais rien (1988), pièce elle-même intégrée à l'installation Les Dix Personnages, inclut l'œuvre Ficelles $(1985)^{30}$. Le fonctionnement de cette genèse perpétuelle est un procédé comparé par l'artiste lui-même au système des "matriochkas ", ou poupées russes ${ }^{31}$.

29 Boris Groys, «Un homme qui veut duper le temps ", op. cit., p. 19.

30 La datation finale des ouvres peut fréquemment changer.

31 Ilya Kabakov, entretien avec Nadine Pouillon, «Le musée : temple ou décharge? », in Ilya Kabakov Installations 1983-1995, op. cit., p. 20. 


\subsection{La narration incarnée}

Les Dix Personnages ${ }^{32}$ intègre l'histoire de dix créations imaginaires, habitants invisibles des chambres d'un appartement communautaire. À travers ces personnages, Kabakov s'offre la liberté de produire des objets différents, de s'exprimer à travers divers media et ainsi peut-être, de réaliser des autoportraits aux visages changeants :

Ce sont dix fables : ils suggèrent dix comportements de l'Homo Sovieticus pour réagir au monde, dix attitudes psychologiques, dix perspectives sur le vide, dix parodies aux traditions esthétiques par lesquelles Kabakov a développé son vocabulaire, dix aspects de la personnalité de Kabakov. ${ }^{33}$

Autour de la trame narrative du couloir, les chambres font figure de portraits. Les personnages, systématiquement absents, sont décrits par des témoignages - du voisin ou de l'inspecteur de police - et par le monde des objets qu'ils ont laissé derrière eux, sanctuaires de la vie privée ouverts à la vue du public. Devant chaque porte ouverte, le spectateur peut lire l'histoire de son in-occupant, arpenter ces espaces mentaux soviétiques. Devant la chambre de L'homme qui s'est envolé dans l'espace, il est décrit que ce dernier était passionné par la conquête de l'espace, qu'il avait recouvert sa chambre d'affiches de propagande car c'était meilleur marché que la tapisserie, et qu'un jour - le 12 avril $1982^{34}$ - il s'était envolé... En effet, un trou au plafond, des gravats au sol et les chaussures qui n'ont pas suivi son envol en témoignent.

32 Les Dix Personnages, installation, 1988.

33 Note sur le site des artistes Ilya et Emilia Kabakov : http://www.ilya-emilia-kabakov.com/ index.php/albums. Traduction personnelle de l'anglais.

34 Yuri Gagarine a effectué la mission qui lui a valu le titre de " premier homme à avoir voyagé dans l'espace » le 12 avril 1961. 


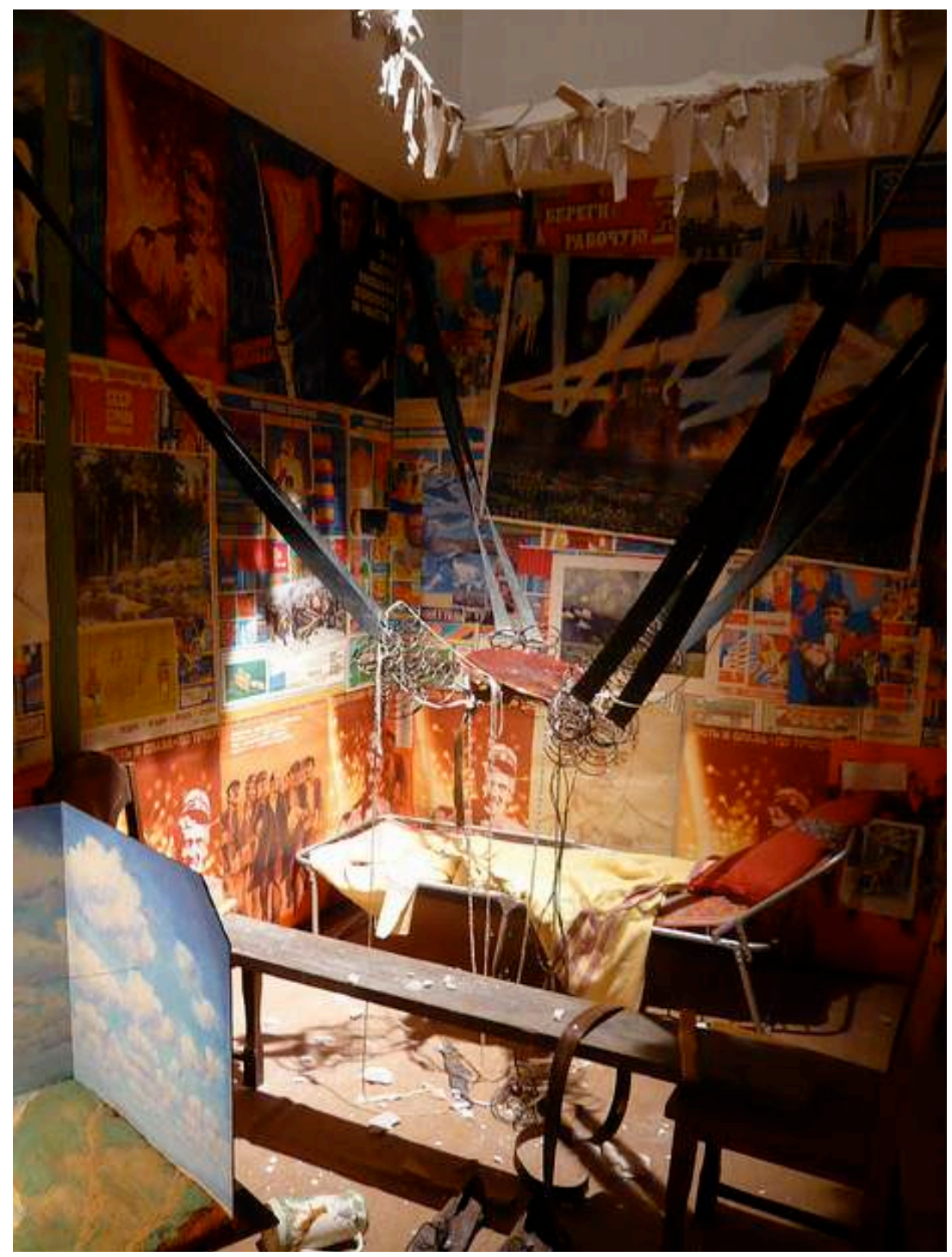

Ilya Kabakov, L'homme qui s'est envolé dans l'espace, 1985.

Ilya Kabakov crée un personnage littéraire, puis active l'imaginaire du spectateur, qui suit sa trajectoire des yeux et se le présente, traversant les différentes strates du plafond et de la toiture, avant de réaliser son rêve d'envol. Grâce à l'interaction avec le regardeur, ce Yuri Gagarine prend vie.

L'environnement recréé ancre dans le réel cette histoire surréaliste : tous les objets, les affiches, le pichet au sol sont chinés dans la vie soviétique et leur imbrication fait surgir du réel et du sensible. Le couloir de l'appartement communautaire intègre un spectateur à la fois acteur et lecteur, et le fait évoluer dans un récit plastique. 
Ilya Kabakov, déjà à ses premières heures conceptualiste moscovite, effectuait un réel travail d'archivage. Il collectait les documents qui concernaient son cercle d'amis (MANI), les reproductions de leurs œuvres, leurs projets, croquis. Il disposait tout en portfolio, dans des boîtes, des caisses. Ses dessins sont parfois représentés sur des paravents, collés, reliés, dépliables jusqu’à cinquante mètres pour le plus long. Ce travail du pli, de l'archivage, de la compilation, de la «mise en boîte " pourrait avoir été influencé par le premier exil de Kabakov enfant, la peur de la censure ne faisant qu'accroître ce sentiment que tout peut disparaître. Il donne le sentiment, à travers ses installations, d'avoir isolé en lui ce monde soviétique voué à l'engloutissement, et de pouvoir le recréer pour donner à voir et à sentir ce qu'avaient pu être l'absurdité, la violence mais aussi la poésie de la vie soviétique.

\subsection{Abstraction des dialogues}

Les textes présents dans l'œuvre de l'artiste sont de plusieurs natures : témoignages fictifs, textes théoriques de l'artiste, récit d'un narrateur omniscient, ou phrases accrochées à un objet.

Parfois interviennent des dialogues, et alors l'absurdité en ressort davantage. Ils apparaissent pour signifier le manque de communication : "À qui est cette tasse ?! », interjection présentée plus haut et inscrite près d'une tasse sur un tableau, évolue en «À qui est ce clou ? ! ", ou "À qui est cette mouche ? ! . Dans un monde où la mise en commun des biens fut idéologique, ce questionnement sur la propriété prend un sens particulier. Tout élément gênant doit être identifié, et son propriétaire doit endosser une responsabilité coupable. La réponse est systématiquement la même : un " Je ne sais pas " libertaire et qui renvoie cette question accusatrice à l'absurdité de sa détermination : il n'y a pas de coupable.

[...] bien souvent, ces réflexions sont tout ce qu'il y a de plus typique de notre univers. Ainsi, par exemple : "Anna Petrovna Sobakina - "Qui a planté ce clou ? Grigori Lvovitch Zimine - Je ne sais pas." " Chez nous, "Je ne sais pas " est la réponse la plus courante, et même les questions commencent par "Vous ne savez pas où se trouve... ? - Je ne sais pas. " "Vous ne savez pas, pour aller... ? - Je ne sais pas. " " À qui est cette râpe ? - Je ne sais pas. »

Pour nous, ce " je ne sais pas " est une réponse éminemment philosophique. Parce que non seulement j'ignore à qui est la râpe, mais je ne sais rien en général : "Et fichez-moi la paix, je vous en supplie ! Je ne sais pas et allez vous faire voir... $»^{35}$

35 Ilya Kabakov, " 52 entretiens dans la cuisine communautaire », dialogues entre Ilya Kabakov et Yuri Kuper, in. Ilya Kabakov Installations 1983-1995, op. cit., p. 142. 
Les phrases apportent sinon du sens, du moins de l'esthétique : les mots n'opèrent pas comme les signifiants d'une idée ou d'un objet concret mais d'un univers, d'une ambiance. Ils sont la partie immergée d'un iceberg soviétique qui aurait échappé au dégel.

Une autre installation, monumentale cette fois, pose la question : « À qui sont ces ailes ? ", en référence aux quatre paires d'ailes en néon disposées sur la façade d'un commissariat de la ville d'Utrecht ${ }^{36}$. Ce questionnement administratif portant sur la propriété d'un élément imaginaire à caractère sacré fait une fois de plus surgir l'absurde au cœur du réel.

Dans La Cuisine communautaire ${ }^{37}$, on peut entendre une conversation entre deux hommes et deux femmes. Si l'on tend l'oreille, on s'aperçoit qu'il ne s'agit pas d'un dialogue mais que ces voix distillent des bribes de phrases, de querelles extraites de cet espace qu'ils doivent partager. Cette pièce octogonale est tapissée de gris, son plafond abrite de hauts murs couverts d'innombrables ustensiles de cuisine, et tout en haut sont disposés 39 tableaux du genre « À qui est cette tasse ?». Le haut de cette cathédrale est tendu de fils auxquels pendent des déchets et des textes. À hauteur de regard sont étendus des dépliants sur lesquels figurent photographies et textes : "Bon, maintenant je vais repasser mon linge..." ", "Vous pouvez le poser, allez-y, je suis en train de ranger...", "Non, nous, dans notre immeuble, on jette les ordures proprement... ", etc.

Malgré la prolifération de mots il n'y a pas de dialogue. Les mots sont utilisés au même titre que les objets, par ce que l'on pourrait qualifier d'un procédé de dé-sémantisation. S'ils constituent des phrases, tout sens profond en est exclu. Il s'agit d'une matérialisation extrême du langage, qui ne renvoie qu'au caractère physique des choses. Il devient alors un matériau permettant, au même titre qu'un objet, l'élaboration d'une essence abstraite. Cela participe de l'installation totale non plus comme mimesis de l'univers soviétique, mais comme mnemosis.

\section{Déviations}

Dans son travail, Kabakov utilise les textes à différentes fins, comme nous l'avons vu. Mais toute la structure de l'installation totale lui permet de ruser, et d'éviter divers écueils : la disparition d'œuvres anciennes ou leur relégation aux archives - par leur réutilisation, ou la limitation à un seul genre artistique - par l'utilisation de divers media. Il utilise également un procédé que l'on ne trouve habituellement pas en art mais en littérature : le recours à la pseudonymie.

36 À qui sont ces ailes?, installation, 1991. Festival Nightlines, Utrecht, Pays-Bas.

37 En exposition permanente au musée Maillol, Fondation Dina Vierny, Paris. 


\subsection{Le refus de l'Histoire}

Le rapport de l'artiste à la narration intervient en opposition à son rapport à l'Histoire. Il tisse un récit permanent, qui intègre des créations anciennes pour les faire perdurer et leur empêcher une mort et un oubli annoncés. Pour utiliser la formule de Boris Groys, il veut "duper le temps " ${ }^{38}$. Dans le terme "soviétique ", il ne faut pas entendre le côté politique mais bien les conséquences de cette politique sur l'ensemble d'objets, de lieux et de personnes qu'elle a influencés ou engendrés. C’est en ce sens que Groys évoque une civilisation :

La civilisation soviétique est la première civilisation de l'histoire moderne qui soit morte : toutes les autres étaient antérieures à la modernité. ${ }^{39}$

L'histoire individuelle et la grande Histoire collective se rejoignent et s'unissent dans ce terme. Ainsi, en inventant des personnages et en recréant les formes de l'univers soviétique, sa disparition progressive n'intervient pas encore, le temps semble figé. Ce travail n'est pas une critique politique, mais un témoignage des formes les plus abstraites de ce monde en suspens : l'absurde, la tension, la fuite, l'imaginaire...

C'est précisément grâce à l'absurdité des situations décrites, à la disparition du possible amenée par le récit, que l'on assiste à l'apparition du sensible.

Dans les années qui ont suivi son émigration aux États-Unis, Kabakov est devenu l'ambassadeur de l'univers archétypal soviétique. En anthropologue averti, il s'est fait le narrateur omniscient des chroniques d'une fin annoncée. Il tend aujourd'hui, avec son épouse Emilia, moins au témoignage de cet univers qu'à des questionnements plus universels.

\subsection{L'auteur est un autre}

[...] Kabakov a adopté une procédure qu'on pourrait décrire comme mode d'auto-expropriation, par lequel il attribue ses propres œuvres à d'autres artistes. Inévitablement, la question est de savoir pourquoi il a besoin de recourir à un jeu si complexe d'anonymat et de pseudonymie, qui peut-être familier dans le monde de la littérature mais reste très rare dans le domaine de l'art. ${ }^{40}$

Charles Rosenthal est le personnage fictif d'un artiste réaliste, créé par Kabakov en $1999^{41}$. Ce personnage est tout-à-fait représentatif du dédoublement, voire de la démultiplication de(s) Kabakov. Il serait le prédécesseur de l'artiste, sa biographie indiquant qu'il vécut en début de siècle, entre 1898 et

38 Boris Groys, «Un homme qui veut duper le temps », op. cit.,p. 17.

39 Ibid., p. 19.

40 Boris Groys, "The Theatre of Authorship ", in Ilya Kabakov : Installations. Catalogue Raisonné, op. cit., t. I, p. 33. Traduction personnelle de l'anglais.

41 La vie et l'ouvre de Charles Rosenthal, installation, 1999. 
1933, menant une vie sans talent particulier, faible en événements personnels marquants. De même que Kabakov, il serait né en Ukraine de parents juifs.

Il est intéressant de noter que cette fiction croise plusieurs réalités historiques, notamment à travers l'école de Marc Chagall, dirigée un temps par Kazimir Malevitch, dans laquelle aurait été formé Charles Rosenthal. Émigré à Paris, capitale artistique de l'époque, des talents de retoucheur acquis pendant son enfance l'aideraient à survivre. Un nouveau parallèle intéressant, puisque c'est grâce au maquillage photographique que Rosenthal survit, alors que Kabakov maquille sa pratique personnelle grâce à l'illustration. Une fois à Paris, cet artiste fictif se concentre sur la reproduction de la Russie socialiste qu'il a quittée. Un accident stoppe net les balbutiements de cette carrière : Charles Rosenthal meurt renversé par une voiture. Ce n'est que bien plus tard que son ouvre sera reconnue.

Dans les faits, ce personnage permet à Kabakov de s'exprimer de manière réaliste, ou d'expérimenter tour à tour le suprématisme, le cubisme, le constructivisme, le réalisme, ou encore l'association de ces différents genres. Il reconstitue à travers lui le chaînon manquant à l'histoire de l'art russe, permettant de faire le lien entre les avant-gardes des années 1920 et le conceptualisme des années $1980^{42}$.

Sergueï Stepanovitch Koshelev est un autre artiste fictif que l'on découvre dans l'installation L'incident au musée ou la musique de l'eau ${ }^{43}$. L'incident en question est l'inondation d'un musée, épanchée tant bien que mal par ses employés qui ont disposé des bâches sur des chaises, des casseroles et des seaux au sol pour récupérer l'eau qui s'écoule du plafond. Une musique aquatique du compositeur Vladimir Tarasov rythme par le bruit des gouttes le parcours du spectateur dans cette exposition sinistrée. Les œuvres présentées sont celles de S. Kochelev, lui aussi de l'école réaliste socialiste. Ce travail apparaît comme une synthèse de divers courants artistiques :

Chacun avait droit à sa place : le réalisme des " ambulants ", le formalisme de Cézanne, le « genrisme " de Menzel, aussi bien que l'" air » de Corot. Il devait en résulter un certain "tableau idéal ", auquel, selon Kochelev, l'histoire de l'art aspirait depuis toujours. ${ }^{44}$

Le « tableau idéal » semble lui aussi sinistré, et laisse la place à la composition de Tarasov qui rend cette œuvre d'art totale. Lorsque Kabakov attribue son travail à un autre c'est en artiste raté, incompris, qu'il tente des innovations formelles aujourd'hui caduques, et plonge dans des écueils rendus intelligibles par la forme absolue de l'installation totale.

42 Alexandre Stroev, « Des histoires alternatives : des projets pour la littérature, l'histoire et l'histoire de l'art ", in Frontières de l'histoire littéraire, sous la direction de Jean Bessière et Judit Maár, L'Harmattan, Paris, 2008, p. 145-155, p. 151.

43 Installation, 1992. Galerie Ronald Feldman, New-York.

44 Ilya Kabakov, in Ilya Kabakov Installations 1983-1995, op. cit., p.168. 
Kabakov, imprégné de la tradition du roman russe, se démultipliait en plusieurs personnages fictifs, à qui il assignait un jeu de rôles. Il justifiait ainsi, dans un contexte artistique qui y était peu préparé, le passage d'une technique à une autre, ses larges emprunts au domaine du théâtre, ainsi que le recours à des pratiques artistiques conventionnelles. ${ }^{45}$

Ainsi, on peut relever de nombreuses occurrences de ces artistes ratés : Charles Rosenthal, Sergueï Kochelev, des « artisans réalistes » anonymes, réalisant de " mauvais produits", tels que L'artiste sans talent (1988), et d'autres à qui l'on doit les peintures figurant dans le installations Le Tableau non accroché (1992), L'Incident dans le couloir, devant la cuisine (1989), Trois Nuits (1989), etc.

Mais tous les alter-ego de Kabakov ne sont pas des artistes, ni même des artisans du réalisme socialiste. Il en invente également d'autres qui ont des occupations désuètes, ou pour qui la peinture relève d'une manie, n'ayant aucune attente envers un regardeur averti : J'aime recouvrir les planches de peinture blanche $(1991)^{46}$ par exemple. Ceci étant dit, la narration est partout indispensable pour différencier l'artiste réel Kabakov de son alter-ego fictif. C'est en ce sens que l'on considère que ce dédoublement, cette auto-expropriation évoquée plus haut par Boris Groys est un procédé de fiction littéraire, permettant à Kabakov de continuer à peindre malgré le manque de talent qu'il s'accorde :

En deux mots, j'ai arrêté de peindre parce que je n'ai pas de talent... Comme j'en étais conscient, j'ai préféré ne pas m'aventurer plus avant sur ce chemin déjà emprunté par tant de véritables génies. ${ }^{47}$

\subsection{Objet réel, objet verbé}

L'utilisation des objets endosse différents rôles dans le travail de Kabakov. Tentons de les définir à travers trois œuvres.

Dans la Cuisine communautaire nous l'avons vu, les objets comme les textes ou les voix, sont les éléments constitutifs d'une entité plus large qui tend à la mnemosis du monde soviétique. Ils apparaissent comme des reliques de ce monde disparu.

Prenons l'exemple de Je plonge dans les souvenirs ${ }^{48}$. Cette pièce, consacrée à la mémoire, a ceci de particulier que tous ses éléments constitutifs ont appartenu à une amie d'Emilia Kabakov, qui les lui a légués. Or, ce fait n'est pas présenté

45 Jean-Hubert Martin, "Kabakov témoin », in Ilya Kabakov Installations 1983-1995, op. cit., p. 12.

46 Pièce de l'installation L'Asile d'aliénés ou l'Institut de recherches créatives, 1991, Malmö, Rooseum.

47 Ilya Kabakov en entretien avec Nadine Pouillon, in Ilya Kabakov Installations 1983-1995, op. cit., p. 24.

48 Partie de l'installation totale La Maison aux Personnages, érigée en 2009 à Bordeaux dans l'espace public et prenant la forme d'une maison de quartier, constituée de sept pièces chacune consacrée à un personnage imaginaire. Le thème de la kommunalka n'est pas prédominant dans cette maison, seule une pièce y fait référence : Je dors dans le jardin. La Maison aux Personnages, 2009, place Amélie Raba Léon, Bordeaux. 
au spectateur. Tous les objets et les meubles exposés sont donc, au sens propre, chargés d'histoire. Des images sont projetées sur un mur de la chambre de l'inconnue. On y retrouve des photographies personnelles de personnages réels, comme les époux Kabakov et la donatrice disparue. La valeur d'authenticité donne à cette pièce une fonction de mémorial, y consignant des souvenirs privés pour une mise en Histoire, publique.

Les objets la constituant ayant un rôle d'intercession symbolique entre réel et imaginaire, on peut considérer que cette pièce est à la fois mythologique et authentique. C'est la valeur subjective - inessentielle - de ces objets qui prime.

La Caisse de Détritus est une œuvre datant de 1990. Une caisse remplie d'objets est posée au sol, et devant elle une chaise invite à découvrir ces objets de plus près. À chaque élément est attachée une étiquette qui, dès que le spectateur s'en saisit, le renvoie à la position de voyeur, voire de voleur, qu'il vient d'adopter : "Lâche ça, conasse, sale pute !", "Remets-ça à sa place, sale con ! ", etc.

Le rapport entre l'espace public du musée, un espace ouvert et partagé, et l'espace privé de la caisse met le spectateur dans une position inconfortable. Il prend soudainement conscience du glissement qui s'opère : les objets tacitement offerts au toucher s'avèrent être la propriété de quelqu'un. Ce sont les objets qui invectivent directement le spectateur, comme s'ils étaient doués de parole ; de détritus ils deviennent le signe, la trace, l'incarnation de la personne à qui ils ont appartenu, qu'elle soit réelle ou fictive.

Dans toute l'œuvre " soviétique " de Kabakov - que nous différencions de son œuvre plus universelle déjà évoquée - l'objet prend successivement le rôle de relique, d'objet de mémoire et d'incarnation du "soviétique ". Il est en interaction permanente avec le verbe, qui lui confère ses différents rôles.

Par toutes ces déviations - Histoire, signature, objet - l'artiste fabrique un univers, et crée son propre "modèle de monde ". Ce monde parallèle, trace mnésique ${ }^{49}$ à la fois artificielle et authentique, permet que les spectateurs expérimentant l'œuvre puissent "non seulement se demander ce qu'avait pu être d'y vivre "ici et maintenant", mais encore pour un bref instant puissent éprouver ces espaces communs et les mystères de cet environnement étranger, comme si c'était le leur $»^{50}$.

49 Nous emprunterons ici ce terme à la psychologie cognitive : « Le terme de trace mnésique a été employé dès le début du $\mathrm{XX}^{\mathrm{e}}$ siècle par les psychologues pour désigner ce qui est conservé par la mémoire. Deux points de vue se sont opposés au sujet de l'interprétation de l'évolution de la trace. Dans la perspective la plus ancienne, la trace mnésique s'affaiblit, perd progressivement les caractéristiques distinctives de la figure originale, sauf celles qui ont été accentuées dans la perception ou renforcées dans la reproduction : c'est l'oubli. Pour les théoriciens de la forme (Gestalt) au contraire, la trace, au lieu de s'affaiblir, se stabilise dans le sens d'une "bonne" forme (aisément mémorisable) par assimilation à un objet ou par accentuation d'un détail. ", Jean-François Richard, Encyclopaedia Universalis, 2012.

50 Robert Storr, «A world apart », in Ilya Kabakov : Installations. Catalogue Raisonne, op. cit., t. II, p. 7. 
La doctrine soviétique tendant à une société idéale, dont la construction passait également par une unification des pratiques créatives dans ce que l'on pourrait qualifier d'œuvre d'art totale - au sens Wagnérien du terme, et dans l'acception qu'en a fait Boris Groys dans sa réflexion sur l'ère stalinienne ${ }^{51}$ nous pouvons considérer qu'llya Kabakov dévie de ce parcours imposé aux sens. Il part non pas de l'idée d'une société soviétique unie, mais des effets psychologiques de cette lutte incessante avec l'idéologie, il se fait narrateur des déviances et des dégénérescences qu'elle a pu engendrer sur ses sujets. Il synthétise les répercussions de cette aspiration à l'idéal sur l'Homo Sovieticus.

Dans les œuvres de Kabakov, la fabrique du soviétique s'est tissée dans une volonté de redessiner les traces de son passé, pour transporter le spectateur dans un ailleurs choisi, littéraire et bientôt littéral. C'est ainsi qu'il a su recréer, pour l'offrir au regard et aux sens, une version de ce qu'avait pu être le vivre ensemble soviétique, dans une kommunalka imaginaire, rendant visibles les traces mnésiques de son monde intérieur.

$\begin{array}{r}\text { Alice Cazaux } \\ \text { Doctorante }\end{array}$
EA 4593 CLARE, Artes
Université Michel de Montaigne Bordeaux 3
CRECOB
alicecazaux@live.fr

\title{
Résumé
}

Ilya Kabakov, qui se qualifie lui-même d'artiste soviétique, dévie du parcours réaliste socialiste imposé aux sens, recréant dans ses installations totales les effets psychologiques d'une lutte incessante avec l'idéologie, et se fait narrateur des déviances et des dégénérescences qu'elle a pu engendrer sur ses sujets. Il synthétise les répercussions de cette aspiration à l'idéal sur l'Homo Sovieticus à travers la représentation des appartements communautaires, les kommunalki.

Mots-clés

Ilya Kabakov, kommunalka, installation totale, soviétique, art contemporain.

\begin{abstract}
Ilya Kabakov, who calls himself Sovietic artist, deviates from the social realistic route imposed to senses. In recreating the psychological effects of such an incessant struggle with ideology in his total installations, he takes place as a storyteller of deviances and degenerations that ideology could lead to his subjects. He synthesizes the impact on the Homo Sovieticus of this aspiration to the ideal, through the representation of communal apartments, the kommunalki.
\end{abstract}

\section{Keywords}

Ilya Kabakov, kommunalka, total installation, soviet art, contemporary art.

51 Boris Groys, Staline ceuvre d'art totale, collection Rayon Art, éditions Jacqueline Chambon, 1988. Traduit du russe par Edith Lalliard. 